\title{
Critical vertices in k-connected Digraphs
}

\section{W. Mader(Hannover)}

DEdicATEd TO THE MEMORY OF MY FRIENd RUdolf HALin

Prof. Dr. W. Mader

Dept. of Mathematics

University of Hanover

Welfengarten 1

$\underline{30167 \text { Hannover }}$

Germany

E-mail:mader@math.uni-hannover.de 


\begin{abstract}
It is proved that every non-complete, finite digraph of connectivity number $k$ has a fragment $F$ containing at most $k$ critical vertices. The following result is a direct consequence: every $k$-connected, finite digraph $D$ of minimum out- and indegree at least $2 k+m-1$ for positive integers $k, m$ has a subdigraph $H$ of minimum outdegree or minimum indegree at least $m-1$ such that $D-x$ is $k$ connected for all $x \in V(H)$. For $m=1$, this implies immediately the existence of a vertex of indgree or outdegree less than $2 k$ in a $k$-critical, finite digraph, which was proved in [17].
\end{abstract}




\section{Introduction}

Rudolf Halin proved in 1969 that every minimally $k$-connected, finite graph contains a vertex of degree $k$ [1]. This important result inspired a lot of further investigations on the existence and number of vertices of small degree in minimally $k$-(vertex-) connected or $k$-edge -connected or critically $k$-connected graphs and digraphs.

First we will explain these concepts more precisely. All graphs and digraphs in this paper are assumed to be finite and do not contain parallel edges ( of the same direction). If parallel edges are allowed, we speak of multigraphs and multidigraphs. A $k$-connected graph (or digraph) is minimally [critically] $k$-connected, if deleting any edge [vertex], the connectivity number becomes less than $k$. For edge-connectivity and for multigraphs and multidigraphs we use corresponding concepts.

A transfer of Halin's result to digraphs was given by T. Kameda in [9], showing that every minimally $k$-connected digraph has a vertex of indegree $k$ or outdegree $k$. This was improved in [13] to the fact that it always contains even a vertex of outdegree $k$. D.R. Lick [7] proved that every minimally $k$-edge connected multigraph has a vertex of degree $k$ and it was shown in [10] that a minimally $k$-edge-connected multidigraph has a vertex of in- and outdgree $k$. Chartrand, Kaugars, and Lick [8] proved that every critically $k$-connected graph has a vertex of degree at most $\frac{3 k-2}{2}$ and I proved in [17] that a critically $k$-connected digraph has a vertex of outdegree or indegree less than $2 k$, but not necessarily always one of outdegree less than $2 k$. The bound in the last two results mentioned is best possible.

Whereas a critically $k$-edge-connected graph has a vertex of degree $k$ by [14], as far as I know, the corresponding concept for digraphs has not been considered until now. But I conjecture that a critically $k$-edge-connected digraph has always a vertex of outdegree or indegree less than $2 k$. This bound would be best possible, as the slightly modified Example (6) in [18] shows.

There are still a lot of open problems, especially on the number of vertices of small degree, considered also by R. Halin himself in [2], [3], [4], [5], and [6]. A survey on results and conjectures on [generalized] multiple criticality of graphs and digraphs is found in [20]. Perhaps the most interesting and fundamental open problem in our context is the following.

Conjecture 1 [12]. Every minimally $k$-connected digraph has a vertex of indegree and outdegree $k$.

The case $k=1$ is the above mentioned result on minimally $k$-edge-connected multidigraphs [10]. The case $k=2$ was proved in [19]. For all $k \geq 3$ the conjecture is open. But it was shown in [19] that for $k \geq 2$, every minimally $k$-connected digraph contains a vertex $z$ with $\min \left\{d^{+}(z), d^{-}(z)\right\}=k$ and 
$\max \left\{d^{+}(z), d^{-}(z)\right\} \leq 2 k-2$.

Before we state the main result of our paper, we should fix the terminology and the notation not so common. For a digraph $D,|D|$ denotes $|V(D)|$ and $x \in D$ means $x \in V(D)$. For subdigraphs $H, G \subseteq D$ and any vertex set $S$, define $H \cap S:=V(H) \cap S$, and instead of $V(H) \cap V(G)=\emptyset$ we write $H \cap G=\emptyset$. We call a digraph connected, if it is "strongly connected" in the usual sense. A path and a circuit in a digraph are always continuously directed. A digraph $D$ is $k$-connected iff $|D| \geq k+1$ and, for every pair $a, b$ of distinct vertices, there are at least $k$ openly disjoint paths from $a$ to $b$. So the complete digraph $\overleftrightarrow{K}_{k+1}$ is $k$-connected but not $(k+1)$-connected, for all integers $k \geq 0$. For such a digraph $D$ we say that it has the connectivity number $k$ and we write $\kappa(D)=k$. A vertex $x$ is critical in $D$ if $\kappa(D-x)<\kappa(D)$, hence $\kappa(D-x)=\kappa(D)-1$ holds. Define $\operatorname{Cr}(D):=\{x \in D: x$ critical in $D\}$ and for $H \subseteq D$ let be $H_{c r}:=H \cap C r(D)$. A digraph $D$ is critically $k$-connected or $k$-critical iff $\kappa(D)=k$ and $C r(D)=V(D)$. A separating set of a digraph $D$ be always an $S \subseteq V(D)$ such that $D-S$ is not (strongly) connected. By Menger's theorem, every non-complete digraph $D$ has a separating set $S$ with $|S|=\kappa(D)$; we call such an $S$ a least separating set. It is easy to see that a vertex is critical in a non-complete digraph $D$ iff it is contained in a least separating set of $D$.

For a subdigraph $H \subseteq D$ (which is always supposed to be not empty) or a subset $H \subseteq V(D)$, let be $N^{+}(H):=\{y \in D: y \notin H$ and there is an $x \in H$ with $(x, y) \in E(D)\}$ the set of outneighbours of $H$ in $D$, and be $N^{-}(D)$ correspondingly the set of inneighbours of $H$ in $D$. For an $x \in D$ we write ( for instance ) $N^{+}(x)$ instead of $N^{+}(\{x\})$. For $x \in D, d^{+}(x):=\left|N^{+}(x)\right|$ and $d^{-}(x):=\left|N^{-}(x)\right|$ denote the outdegree and indegree of $x$, respectively. The minimum outdegree and the minimum indegree of a digraph $D$ are denoted by $\delta^{+}(D)$ and $\delta^{-}(D)$, respectively; furthermore, $\delta(D):=\min \left\{\delta^{+}(D), \delta^{-}(D)\right\}$. An induced subgraph $H$ of $D$ is a positive fragment iff $D-\left(V(H) \cup N^{+}(H)\right) \neq \emptyset$ and $\left|N^{+}(H)\right|=\kappa(D)$ hold. Correspondingly, we define a negative fragment, and a fragment is a positive or negative fragment. Also without mentioning the sign, we consider a fragment $F$ always with a certain sign, but sometimes we write $\operatorname{sgn} F$ for it. A subdigraph $H$ can be a positive and at the same time a negative fragment, but we consider these fragments different. If $F$ is a positive [ negative ] fragment of $D$, then $\bar{F}$ denotes the negative [positive] fragment $D-\left(V(F) \cup N^{+}(F)\right)\left[D-\left(V(F) \cup N^{-}(F)\right)\right]$. Obviously, every non-complete digraph has a positive fragment and a negative fragment.

Now we can state our main result.

Theorem 1: Every non-complete, finite digraph $D$ has a positive or a negative fragment $F$ with $|F \cap C r(D)| \leq \kappa(D)$.

The proof of this theorem is found in section 3. First we will give some consequences and examples, and will compare the directed case with the undirected one. The following result is an immediate consequence of Theorem 1.

Corollary 1 [17]: Every k-critical non - complete digraph has a positive or 
a negative fragment $F$ with $|F| \leq k$. Hence every $k$-critical digraph has a vertex $x$ of $d^{+}(x)<2 k$ or $d^{-}(x)<2 k$

Originally, I was not especially interested in finding an easier proof for this result, but I hoped to get a generalization of the following result to digraphs.

Theorem 2 [21]: Let $G$ be a k-connected graph with $\delta(G) \geq 2(k-1+$ $m)^{2}+m-1$ for positive integers $k, m$. Then there is a (non-empty) subgraph $H \subseteq G$ with $\delta(H) \geq m-1$ such that $\kappa(G-A) \geq k$ for all $A \subseteq V(H)$ with $|A| \leq m$.

However, we have only succeeded in proving such a result for $|A|=1$ for digraphs.

Corollary 2: Every $k$-connected digraph $D$ with $\delta(D) \geq 2 k+m-1$ for positive integers $k, m$ has a (non-empty) subdigraph $H$ with $\delta^{+}(H) \geq m-1$ or $\delta^{-}(H) \geq m-1$ such that $\kappa(D-x) \geq k$ holds for all $x \in H$.

Proof: It is obvious for $\kappa(D)>k$. So we may assume $D$ non-complete and $\kappa(D)=k$. Then Theorem 1 delivers a positive or negative fragment $F$ with $|F \cap C r(D)| \leq k$. But $\delta(D) \geq 2 k+m-1$ implies $\delta^{\operatorname{sgn} F}(F) \geq k+m-1$ and therefore, $|F| \geq k+m$. Then $H:=F-C r(D)$ has the asserted properties -

For $m=1$, this is again the result mentioned in the second half of Corollary 1. For $m=2$, it says that in a $k$-connected digraph $D$ with $\delta(D) \geq 2 k+1$ there is a circuit $C$ such that deleting any $x \in C$ from $D$ maintains connectivity $k$.

It was shown in [15] that every non-complete graph $G$ of connectivity number $k$ has a fragment $F$ with $F \cap C r(G)=\emptyset$ or a fragment $F$ with $V(F) \subseteq C r(G)$ and $|F| \leq \frac{k}{2}$. In the directed case, all fragments of a digraph $D$ with $\kappa(D)=k$ can be arbitrarily large ( for fixed $k$ ) in spite of $|F \cap C r(D)| \geq k$ for all fragments $F$ of $D$. Since it is known from [17] and [18] that the results of Corollary 1 are best possible, there cannot be in Theorem 1 always a positive or a negative fragment $F$ with $|F \cap C r(D)|<k$. Example 1 shows once again that $|F \cap C r(D)| \leq k$ and also the lower bound $2 k+m-1$ in Corollary 2 are best possible.

Example 1. Let $K^{1}, \ldots, K^{n}$ be disjoint complete digraphs of order at least $2 k$ and let $S_{i}^{1}, S_{i}^{2}$ be disjoint $k$-element subsets of $V\left(K^{i}\right)$ for $i=1, \ldots, n$. The digraph $D$ arises from $\bigcup_{i=1}^{n} K^{i}$ by addition of all edges from $S_{i}^{1}$ to $S_{i+1}^{2}$ for $i=1, \ldots, n(\bmod \mathrm{n})$.

Then $\kappa(D)=k$ holds, the minimal positive [negative ] fragments are the subgraphs $K^{i}-S_{i}^{1}\left[K^{i}-S_{i}^{2}\right]$ for $i=1, \ldots, n$. So $\operatorname{Cr}(D)=\bigcup_{i=1}^{n}\left(S_{i}^{1} \cup S_{i}^{2}\right)$ holds and for every minimal fragment $F$ we have $|F \cap C r(D)|=k$. If we take all $K^{i}$ of order $2 k, D$ is $k$-critical and has no vertex of indegree or outdegree less than $2 k-1$. If we take all $K^{i}$ of order exceeding $2 k$, then there is no fragment $F$ with $V(F) \subseteq C r(D)$, and we can make $\delta(D)$ arbitrarily large. (This is in contrast to the undirected case, mentioned in the paragraph before Example 1.) If we choose all $K^{i}$ of order $2 k+m-1$, then we have $\delta(D)=2 k+m-2$, but there is no subdigraph $H$ with $\delta^{+}(H) \geq m-1$ or $\delta^{-}(H) \geq m-1$ and with 
$H \cap C r(D)=\emptyset$. So the lower bound $2 k+m-1$ in Corollary 2 is best possible.

We know from [17] and [18] that in a $k$-critical digraph with $k \geq 2$, in general, there need not be a vertex $x$ with $d^{+}(x)<2 k$, hence no positive fragment $F$ with $|F| \leq k$. Therefore, also in Theorem 1, we cannot prescribe the sign of the fragment $F$ ( as also shown in Example 2). This suggests that the same holds for $H$ in Corollary 2. The following example shows that this is true, if $\delta^{-}(H) \geq 2$.

Example 2. Let $a>k$ be positive integers. Let $K$ be a complete digraph with $|K|>a+k$. For $i=1, \ldots, n\left(n \geq \frac{|K|}{k}\right)$ choose a $k$-element vertex set $C_{i}$ of $K$, such that $V(K) \subseteq \bigcup_{i=1}^{n} C_{i}$ holds. For $i=1, \ldots, n$ let $D_{i}=\left(A_{i}, B_{i}\right)$ be a digraph with $V\left(D_{i}\right)=A_{i} \cup B_{i}, A_{i}$ and $B_{i}$ are disjoint, and $\left|A_{i}\right|=a,\left|B_{i}\right|=a^{2}$. Let $E\left(D_{i}\right)$ consist of all edges from $B_{i}$ to $A_{i}$ and from every vertex of $A_{i}$ to exactly $a$ vertices of $B_{i}$ so that every vertex of $B_{i}$ gets indegree 1 . (Hence $D_{i}$ is a directed bipartite multigraph.) Assume that $V(K), V\left(D_{1}\right), \ldots, V\left(D_{n}\right)$ are disjoint. Choose $C_{i}^{\prime} \subseteq V(K)$ of at least $a+k-1$ vertices for $i=1, \ldots, n$. Let $D$ arise from $K \cup \bigcup_{i=1}^{n} D_{i}$ by addition of all edges from $D_{i}$ to $C_{i}$ and from $C_{i}^{\prime}$ to $B_{i}$ for $i=1, \ldots, n$.

Then $\kappa(D)=k$ and $C r(D)=V(K)$. Furthermore, $\delta(D)=a+k$ holds, but there is no negative fragment $F$ with $|F \cap C r(D)| \leq k$, since a negative fragment contains $V(K)-V\left(C_{i}\right)$ for a certain $i$. ( For $k=1$, this is in contrast to 1-critical digraphs: these have a positive and a negative fragment of order 1 , i.e. a vertex of outdegree 1 and a vertex of indegree 1 (see [16] or [18]).) Since all $x \in \bigcup_{i=1}^{n} B_{i}$ have indegree 1 in $D-V(K)$, there is no $H \subseteq D-V(K)$ with $\delta^{-}(H) \geq 2$.

The next example proves that for $k$-critical digraphs $D$ with fixed $k \geq 2$, $\delta^{-}(D)$ can be even arbitrarily large. ( This is quite different from minimally $k$ connected digraphs, which have a vertex of indegree $k$ and a vertex of outdegree $k$, as proved in [13]. ) Furthermore, it shows that in Corollary 2, high indegree is not sufficient for the existence of a subdigraph $H$ of large minimum outdegree or minimum indegree not containing vertices of $\mathrm{Cr}(D)$.

Example 3: Let us take in Example 2 all $D_{i} \cong \stackrel{\leftrightarrow}{K}_{k+m-1}$ for any positive integers $k, m$, but the other notation $K, C_{i}, C_{i}^{\prime}, a$ remain as there. Let $D$ arise from $K \cup \bigcup_{i=1}^{n} D_{i}$ by addition of $k$ disjoint edges from $D_{i}$ to $C_{i}$ for $\mathrm{i}=1, \ldots, \mathrm{n}$ and of all edges from $C_{i}^{\prime}$ to $D_{i}$.

Then $\kappa(D)=k, \delta^{-}(D) \geq a+k-1, C r(D) \supseteq V(K)$, and for $k \geq 2$ as for $m \geq 2,\left|C r(D) \cap D_{i}\right|=k$ holds for $\mathrm{i}=1, \ldots, \mathrm{n}$. For $m=1$ and $k \geq 2, D$ is $k$-critical, but changing the value of the integer $a$, we can make $\delta^{-}(D)$ arbitrarily large. Therefore, for fixed $k \geq 2$, there is no upper bound for $\delta^{-}(D)$ for all $k$-critical digraphs $D$ (dependent only on $k$ ). For $a \geq m \geq 2, \delta^{+}(D)=k+m-2$ and $\left.D_{i}-C r(D)\right) \cong \overleftrightarrow{K}_{m-1}$, and this does not change, if we enlarge $a$, and in this way $\delta^{-}(D)$. So we cannot find an $H \subseteq D-C r(D)$ with bigger minimum outor indegree by enlarging only the minimum indegree of $D$. 
I conjectured in [18] that every $k$-critical digraph has at least two different minimal fragments ( different at least by their sign) with $|F| \leq k$, and similar for vertices of in- or outdegree less than $2 k$. This conjecture is unproved and I believe it is only a special case of the following more general conjecture.

Conjecture 2. Every non-complete digraph $D$ with $\kappa(D)=k$ has at least two different minimal fragments $F$ with $|F \cap C r(D)| \leq k$.

The existence of two such fragments would be best possible, also for arbitrarily large vertex number. For $k \geq 2$, this is shown by the Examples 5 and 6 in [18]. Simple examples for all $k \geq 1$ are digraphs $D$ with exactly one least separating set $S$ and the property that the components of $D-S$ form a chain.

The following conjecture also remains open for all $(k, l, m)$ with $l>1$ and $m>1$.

Conjecture 3. For positive integers $k, l, m$, there is a least integer $f_{k}(l, m)$ with the property that for all $k$-connected digraphs with $\delta(D) \geq 2 k+f_{k}(l, m)$ there is a non-empty subdigraph $H$ with $\delta^{+}(H) \geq m-1$ or $\delta^{-}(H) \geq m-1$ such that $\kappa(D-A) \geq k$ holds for all $A \subseteq V(H)$ with $|A| \leq l$.

Since there are $k$-critical digraphs $D$ with $\delta(D)=2 k-1$ for every positive integer $k$ such that $\kappa(D-A)<k$ for every non-empty $A \subset V(D)$ (e.g. we get some from Example 1, if we take there $K^{i} \cong \overleftrightarrow{K}_{k}$ and $S_{i}^{1}=S_{i}^{2}$ for i=1,., n ), we must have $f_{k}(l, m) \geq 0$, if it exists. For $l=1$, Corollary 2 together with Example 1 shows $f_{k}(1, m)=m-1$ for positive $k$ and $m$. Example 2 also shows that $H$ in Conjecture 3 cannot be found always with $\delta^{-}(H) \geq m-1 \geq 2$ or with prescribed indegrees for special vertices. For instance, any subdigraph $H$ containing vertices of indegree at least 2 having odd distance in the (undirected) multigraph underlying $\mathrm{H}$, in general, cannot be found in $D-C r(D)$, even for arbitrarily large $\delta(D)$.

This shows that a generalization of Proposition 1 in [21], suggested after the Example on page 328 in [21], is not possible. That Proposition says that every connected digraph $D$ with $\delta(D) \geq 2 m$ for a positive integer $m$ contains a path $\mathrm{P}$ of order $m$ such that $D-V(P)$ is connected. I asked there, if a similar result holds for every orientation of an undirected path. Example 2 shows that this is not the case, for instance, for the orientation $P_{0}$ of an undirected path $P$ of length 5 , which has two vertices of indegree 2 of distance 3 on $P$. For given $k \geq 1$, not every digraph $D$ of connectivity number $k$ and high $\delta(D)$ does contain a subdigraph $P_{0} \subseteq D-C r(D)$. ( Hence, for such a $D$ for $k=1$, $D-V\left(P_{0}\right)$ is unconnected for every $P_{0} \subseteq D$, if $\delta(D) \geq 6$. )

\section{Further Notation and some Lemmas}

For the remainder of the paper, $D$ denotes always a finite, non-complete, simple digraph of connectivity number $\kappa(D)=k \geq 1$. Fragments $F_{1}$ and $F_{2}$ 
are crossing, if $s g n F_{1}=\operatorname{sgn} F_{2}, F_{1} \cap F_{2} \neq \emptyset$, and $V\left(F_{1} \cap F_{2}\right) \subset V\left(F_{i}\right)$ for $i=1$, 2 hold. The set of all fragments of $D$ is denoted by $\mathcal{F}(D)$. For any $\mathcal{S} \subseteq \mathcal{F}(D)$, $F \subseteq D$, and $s \in\{+,-\}$ define $\mathcal{S}(F):=\left\{F^{\prime} \in \mathcal{S}: F^{\prime} \subseteq F\right\}, \dot{\mathcal{S}}(F):=\mathcal{S}(F)-\{F\}$, $\overline{\mathcal{S}}:=\bigcup_{F^{\prime} \in \mathcal{S}} V\left(F^{\prime}\right)$ and $\mathcal{S}^{s}:=\{F \in \mathcal{S}: \operatorname{sgn} F=s\}$. For instance, $\mathcal{F}^{+}(D):=$ $\mathcal{F}(D)^{+}$is the set of all positive fragments of $D . F$ is a minimal positive fragment of $D$ means that $F$ is minimal in $\left(\mathcal{F}^{+}(D), \subseteq\right)$, and it does not mean that it is a minimal element of $(\mathcal{F}, \subseteq)$, which is positive. For $F^{\prime}, F$ from $\mathcal{F}(D)$ with $\operatorname{sgn} F^{\prime}=-\operatorname{sgn} F$, we define $F^{\prime} \leq F$ to mean $V\left(F^{\prime}\right) \subseteq V(F) \cup N^{\operatorname{sgn} F}(F)$. Note that in general $F^{\prime} \leq F$ does not imply $\left|F^{\prime}\right| \leq|F|$. Of course, $F^{\prime} \leq F$ holds iff $F^{\prime} \cap \bar{F}=\emptyset$. We will call an $F \in \mathcal{F}(D)$ good, if there is an $F^{\prime} \in \mathcal{F}^{-\operatorname{sgn} F}(D)$ with $F^{\prime} \leq F$. Let $\mathcal{G}(D)$ be the set of all good fragments of $D$. For $H \subseteq D$, we have defined $H_{c r}=H \cap C r(D)$.

If $W$ is a path from a to b, we use $W(a, b]$ and so on in the same way as for intervals; for instance, $W[a, b)$ means the path $W-b$. For a subdigraph or a set of vertices $H$ and an $a \in D-H$, an $a, H$-fan of order $k$ is a set of $a, H$-paths $W_{1}, \ldots, W_{k}$ in $D$ so that the paths $W_{i}-a$ are disjoint and $W_{i} \cap H$ consists exactly of the endvertex of $W_{i}$ for $\mathrm{i}=1, \ldots, \mathrm{k}$. An $H, a$-fan is defined in an analogous way. For an $H \subseteq D$ or $H \subseteq V(D), D(H)$ is the subgraph of $D$ induced by $H$. In formulas, $\cap$ ties stronger than $\cup$, for instance, $A \cup B \cap C$ means $A \cup(B \cap C)$.

The first lemma is well-known, easily proved, and found in many papers, e.g. as Lemme on p. 1254 in [11] or Lemma 1 on p. 266 in [17]. We omit the proof.

Lemma 1. Let $F_{1}, F_{2} \in \mathcal{F}^{+}(D)$ be with $F_{1} \cap F_{2} \neq \emptyset$. Then the following statements are true.

(a) $\left|N^{+}\left(F_{2}\right) \cap F_{1}\right| \geq\left|N^{+}\left(F_{1}\right) \cap \overline{F_{2}}\right|$ and $\left|N^{+}\left(F_{1}\right) \cap F_{2}\right| \geq\left|N^{+}\left(F_{2}\right) \cap \overline{F_{1}}\right|$.

(b) If also $\overline{F_{1}} \cap \overline{F_{2}} \neq \emptyset$, then $F_{1} \cap F_{2}$ is a positive fragment with

$N^{+}\left(F_{1} \cap F_{2}\right)=N^{+}\left(F_{2}\right) \cap F_{1} \cup N^{+}\left(F_{1}\right) \cap N^{+}\left(F_{2}\right) \cup N^{+}\left(F_{1}\right) \cap F_{2}$

and $\overline{F_{1}} \cap \overline{F_{2}}$ is a negative fragment with

$N^{-}\left(\overline{F_{1}} \cap \overline{F_{2}}\right)=N^{+}\left(F_{1}\right) \cap \overline{F_{2}} \cup N^{+}\left(F_{1}\right) \cap N^{+}\left(F_{2}\right) \cup N^{+}\left(F_{2}\right) \cap \overline{F_{1}}$.

Furthermore, $\overline{\overline{F_{1}} \cap \overline{F_{2}}}=D\left(F_{1} \cup F_{2}\right)$ holds.

The good fragments play an essential role in our proof. It follows easily from the definition, that $\mathcal{G}(D)$ is not empty, if and only if there are two disjoint fragments of the same sign. We give an easy criterion for $\mathcal{G}(D) \neq \emptyset$.

Lemma 2. If for an $s \in\{+,-\}$ there are $C \in \mathcal{F}^{s}(D)$ and $B$ minimal in $\left(\mathcal{F}^{s}(D), \subseteq\right)$ with $N^{s}(C) \cap B \neq \emptyset$, then $\mathcal{G}(D) \neq \emptyset$ holds.

Proof. Say, $C$ and $B$ are positive fragments as above. If $C \cap B=\emptyset$, then $B \leq \bar{C}$ and $C \leq \bar{B}$, hence $\{\bar{B}, \bar{C}\} \subseteq \mathcal{G}(D)$. So we may assume $C \cap B \neq \emptyset$. Then we see, $\bar{C} \cap \bar{B}=\emptyset$, since otherwise $B \cap C \subset B$ would be in $\mathcal{F}^{+}(D)$ by Lemma 1 (b), but $B$ is minimal. Hence $\{B, C\} \subseteq \mathcal{G}(D)$ follows as above.

Lemma 3. Let be $A, B_{1}, B_{2} \in \mathcal{F}^{+}(D)$ with $B_{i} \leq \bar{A}$ for $i=1$,2 and $B_{1} \cap B_{2} \neq$ $\emptyset$. Then $\left|\left(N^{+}\left(B_{1}\right) \cup N^{+}\left(B_{2}\right)\right) \cap A\right| \leq k$ holds. 
Proof. We may assume $|A|>k$. By hypothesis, $b \in B_{1} \cap B_{2}$ exists. The inequality $|A|>k$ and $\kappa(D)=k$ imply the existence of an $a \in A-N^{+}\left(B_{1}\right)$. Since $D$ is $k$-connected, there are $k$ openly disjoint paths $W_{1}, \ldots, W_{k}$ from $b$ to $a$. Since $b \in B_{1} \leq \bar{A}$ and $a \in A-\left(N^{+}\left(B_{1}\right) \cup B_{1}\right)$, for every $\mathrm{i}=1, \ldots, \mathrm{k}$, there is exactly one $t_{i} \in N^{+}\left(B_{1}\right) \cap W_{i} \subseteq W_{i}(b, a)$. Then $W_{i}\left[b, t_{i}\right) \subseteq B_{1}$. We now prove the following.

Claim. Let $i \leq k$ be a positive integer. Then

$$
\begin{aligned}
& \text { (a) } W_{i}(b, a) \cap\left(\left(N^{+}\left(B_{1}\right) \cup N^{+}\left(B_{2}\right)\right)-A\right) \neq \emptyset \text { or } \\
& \text { (b) } W_{i}(b, a) \cap N^{+}\left(B_{1}\right) \cap N^{+}\left(B_{2}\right) \neq \emptyset \text { holds. }
\end{aligned}
$$

Proof of the Claim. If $W_{i}(b, a) \cap N^{+}\left(B_{2}\right)=\emptyset$, then $b \in B_{2}$ implies $W_{i}(b, a) \subseteq$ $B_{2}$, hence $t_{i} \in B_{2}$. So $(a)$ holds, since $B_{2} \cap A=\emptyset$.

If $W_{i}(b, a) \cap N^{+}\left(B_{2}\right) \neq \emptyset$, let $y$ be the first vertex of $N^{+}\left(B_{2}\right)$ on the path $W_{i}(b, a)$. If $y=t_{i}$, then $(b)$ holds. So we may assume $y \neq t_{i}$. Then $W_{i}(b, y) \subseteq B_{2}$ and $W_{i}\left(b, t_{i}\right) \subseteq B_{1}$, hence $t_{i} \in B_{2}$ or $y \in B_{1}$ holds. Therefore, $t_{i}$ or $y$ is in $\left(N^{+}\left(B_{1}\right) \cup N^{+}\left(B_{2}\right)\right)-A$, since $B_{j} \cap A=\emptyset$. Hence $(a)$ holds.

If $k_{1}$ is the number of the paths $W_{i}(b, a)$ with property $(b)$, then $\left|\left(N^{+}\left(B_{1}\right) \cup N^{+}\left(B_{2}\right)\right) \cap A\right| \leq\left(2 k-k_{1}\right)-\left(k-k_{1}\right)=k$, since $W_{i}(b, a) \cap W_{j}(b, a)=\emptyset$ for $i \neq j$.

Remark. One could also use Lemma 1(a) for the proof.

We now combine Lemma 1(b) and Lemma 3 into the form in which we will use it.

Lemma 4. Let be $A \in \mathcal{F}^{+}(D)$ with $|A|>k$ and $B_{1}, B_{2} \in \mathcal{F}^{+}(D)$ with $B_{i} \leq \bar{A}$ for $i=1$, 2. If $B_{1} \cap B_{2} \neq \emptyset$, then $B_{1} \cap B_{2} \leq \bar{A}$ and $\overline{\overline{B_{1}} \cap \overline{B_{2}}}=$ $D\left(B_{1} \cup B_{2}\right) \leq \bar{A}$ are positive fragments.

Furthermore, $N^{+}\left(B_{1} \cap B_{2}\right)=N^{+}\left(B_{1}\right) \cap B_{2} \cup N^{+}\left(B_{1}\right) \cap N^{+}\left(B_{2}\right) \cup N^{+}\left(B_{2}\right) \cap B_{1}$ and $N^{+}\left(B_{1} \cup B_{2}\right)=N^{+}\left(B_{1}\right) \cap \overline{B_{2}} \cup N^{+}\left(B_{1}\right) \cap N^{+}\left(B_{2}\right) \cup N^{+}\left(B_{2}\right) \cap \overline{B_{1}}$ hold.

Proof. Since $B_{i} \cap A=\emptyset$ for $i=1,2$ and $|A|>k$, Lemma 3 implies $\overline{B_{1}} \cap \overline{B_{2}} \neq$ $\emptyset$. Then Lemma 4 follows from Lemma $1(\mathrm{~b})$.

\section{Proof of Theorem 1}

Remember that $D$ is always a finite, non-complete,simple digraph of connectivity number $k \geq 1$. We presuppose now further $\left|F_{c r}\right|:=|F \cap C r(D)|>k$ for all $F \in \mathcal{F}(D)$. Since $\mathcal{F}(D) \neq \emptyset$ contains a minimal fragment $B$ and every fragment contains critical vertices by assumption, also $\mathcal{G}(D) \neq \emptyset$ holds by Lemma 2. If there is an $F \in \mathcal{G}(D)$, which is minimal in $\left(\mathcal{F}^{\operatorname{sgn} F}(D), \subseteq\right)$, then choose such an $F$. Otherwise choose an $F$ minimal in $\left(\mathcal{G}^{+}(D), \subseteq\right)$ or in $\left(\mathcal{G}^{-}(D), \subseteq\right)$. Say, we have chosen in this way $\bar{A} \in \mathcal{G}^{-}(D)$ for an $A \in \mathcal{F}^{+}(D)$. Since $\bar{A}$ is good, the set $\mathcal{B}:=\left\{B \in \mathcal{F}^{+}(D): B \leq \bar{A}\right\}$ is not empty, so neither is $\mathcal{B}_{0}:=\{B: B$ 
minimal in $(\mathcal{B}, \subseteq)\}$.

In this whole section $\bar{A}$ is chosen as above.

Consider $B \in \mathcal{B}_{0}$. Since we have assumed $\left|B_{c r}\right|>k, B_{c r}-N^{+}(A) \neq \emptyset$ holds. Hence there is a fragment $C \in \mathcal{F}^{+}(D)$ with $N^{+}(C) \cap\left(B-N^{+}(A)\right) \neq \emptyset$. We will show (1) $C \cap B=\emptyset$ and (2) $C \leq \bar{A}$, i.e. $C \in \mathcal{B}$.

(1) Let be $B \in \mathcal{B}_{0}$ and $C \in \mathcal{F}^{+}(D)$ with $N^{+}(C) \cap\left(B-N^{+}(A)\right) \neq \emptyset$. Then $C \cap B=\emptyset$ holds.

Proof. We assume $C \cap B \neq \emptyset$ and deduce a contradiction to our choice of $\bar{A}, B$. We prove some properties.

$$
\text { (a) } \bar{C} \cap \bar{B}=\emptyset \text {. }
$$

If we had $\bar{C} \cap \bar{B} \neq \emptyset$, then by Lemma 1 (b), $C \cap B$ were a positive fragment, which were properly contained in $B$, since $N^{+}(C) \cap B \neq \emptyset$. But $B$ was chosen minimal positive.

Since $B \leq \bar{A}$ means $A \cap B=\emptyset$, (a) implies

Next we show

$$
\text { (b) } A \cap \bar{C} \subseteq N^{+}(B) \text {. }
$$

$$
\text { (c) } A \cap C \neq \emptyset \text {. }
$$

We assume $A \cap C=\emptyset$, i.e. $C \leq \bar{A}$. Since $|A| \geq\left|A_{c r}\right|>k$, we can apply Lemma 4 to $C, B$. This says that $C \cap B$ is a positive fragment. But this is properly contained in the minimal positive fragment $B$, since $N^{+}(C) \cap B \neq \emptyset$. So (c) follows.

The last property we need is

$$
\text { (d) } \bar{A} \cap \bar{C} \neq \emptyset \text {. }
$$

Let us assume $\bar{A} \cap \bar{C}=\emptyset$. Using (b), we get

$$
V(\bar{C})=\bar{C} \cap N^{+}(B) \cup \bar{C} \cap N^{+}(A) .
$$

Using (c), Lemma 1(a) implies

$$
\left|N^{+}(C) \cap A\right| \geq\left|N^{+}(A) \cap \bar{C}\right|
$$

and using our main assumption $C \cap B \neq \emptyset$

$$
\left|N^{+}(C) \cap B\right| \geq\left|N^{+}(B) \cap \bar{C}\right| .
$$

Using the last two inequalities and the last equality we get

$$
|\bar{C}| \leq\left|N^{+}(C) \cap B\right|+\left|N^{+}(C) \cap A\right| \leq\left|N^{+}(C)\right|=k,
$$

since $A \cap B=\emptyset$. This contradiction to our preassumption on $D$ proves (d).

Lemma 1(b) applied to (c) and (d), shows $\bar{A} \cap \bar{C} \in \mathcal{F}^{-}(D)$. Since $\emptyset \neq$ $N^{+}(C) \cap\left(B-N^{+}(A)\right) \subseteq \bar{A}$, the negative fragment $\bar{A} \cap \bar{C}$ is properly contained in $\bar{A}$. Hence, $\bar{A}$ is not minimal in $\left(\mathcal{F}^{-}(D), \subseteq\right)$. On the other hand, $\bar{A} \cap \bar{C} \leq B$ holds by (a), hence $B \in \mathcal{G}(D)$. Since $B$ is minimal in $\left(\mathcal{F}^{+}(D), \subseteq\right)$, but $\bar{A}$ is not minimal in $\left(\mathcal{F}^{-}(D), \subseteq\right)$, this contradicts our choice of $\bar{A}$. 
(2) Let be $B \in \mathcal{B}_{0}$ and $C \in \mathcal{F}^{+}(D)$ with $N^{+}(C) \cap\left(B-N^{+}(A)\right) \neq \emptyset$. Then $C \in \mathcal{B}$ holds.

Proof. Let us assume $C \cap A \neq \emptyset$. By (1), we know $C \cap B=\emptyset$. Together with $B \leq \bar{A}$, this implies

$$
\text { (a) } B \subseteq\left(\bar{A} \cup N^{+}(A)\right) \cap\left(\bar{C} \cup N^{+}(C)\right)=\bar{A} \cap \bar{C} \cup T
$$

with $T:=\bar{A} \cap N^{+}(C) \cup N^{+}(A) \cap\left(\bar{C} \cup N^{+}(C)\right)$.

Our assumption $C \cap A \neq \emptyset$ and Lemma 1(a) imply $\left|N^{+}(C) \cap \bar{A}\right| \leq\left|N^{+}(A) \cap C\right|$, hence $|T| \leq\left|N^{+}(A)\right|=k$. So, $\bar{A} \cap \bar{C} \neq \emptyset$ follows from (a), since $|B|>k$. Lemma 1(b) says now that $\bar{A} \cap \bar{C} \in \mathcal{F}^{-}(D)$ with $N^{-}(\bar{A} \cap \bar{C})=T$. By (a), so $B \leq \bar{A} \cap \bar{C}$, hence $\bar{A} \cap \bar{C} \in \mathcal{G}^{-}(D)$. But this contradicts the choice of $\bar{A}$, since $\bar{A} \cap \bar{C}$ is properly contained in $\bar{A}$ because $N^{+}(C) \cap\left(B-N^{+}(A)\right) \neq \emptyset$.

For $B_{1}, B_{2} \in \mathcal{B}, B_{1} \cap B_{2} \neq \emptyset$ implies by Lemma 4 that $B_{1} \cap B_{2}$ and $\overline{B_{1}} \cap \overline{B_{2}}$ are fragments of $D$. Therefore, for $B_{1} \in \mathcal{B}_{0}$ and $B_{2} \in \mathcal{B}$ with $B_{1} \cap B_{2} \neq \emptyset$ we have $B_{1} \subseteq B_{2}$; in particular, the elements of $\mathcal{B}_{0}$ are disjoint. For every $B \in \mathcal{B}_{0}$ and $x \in B_{c r}^{*}:=B_{c r}-N^{+}(A) \neq \emptyset$, there is a $C \in \mathcal{F}^{+}(D)$ with $x \in N^{+}(C)$, and by (2) we have $C \in \mathcal{B}$. Hence,

$$
\text { (3) } \bigcup_{C \in \mathcal{B}} N^{+}(C) \supseteq \bigcup_{B \in \mathcal{B}_{0}} B_{c r}^{*}=: S_{0} \text {. }
$$

Since all $B \in \mathcal{B}_{0}$ are disjoint, we know

by assumption on $D$.

$$
\text { (4) }\left|S_{0}\right|=\sum_{B \in \mathcal{B}_{0}}\left|B_{c r}^{*}\right| \geq\left(\sum_{B \in \mathcal{B}_{0}}\left|B_{c r}\right|\right)-\left|N^{+}(A)\right| \geq\left|\mathcal{B}_{0}\right|(k+1)-k
$$

We will show that (3) and (4) are contradictory.

First we construct a subset $\mathcal{C} \subseteq \mathcal{B}$ without crossing elements, but with $\bigcup_{B \in \mathcal{C}} N^{+}(B) \supseteq \bigcup_{B \in \mathcal{B}_{0}} B_{c r}^{*}$. We get such a subset $\mathcal{C} \subseteq \mathcal{B}$ by applying successively the following procedure: if there are in our subset, which we have obtained after some steps, crossing fragments $B_{1} \neq B_{2}$ then replace $B_{1}$ and $B_{2}$ by $B_{1} \cap B_{2}$ and $D\left(B_{1} \cup B_{2}\right)$. This gives again a subset $\mathcal{B}^{\prime} \subseteq \mathcal{B}$, since $B_{1} \cap B_{2}$ and $D\left(B_{1} \cup B_{2}\right)$ are in $\mathcal{B}$ by Lemma 4 . By Lemma 4 again, we have $N^{+}\left(B_{1} \cap B_{2}\right) \cup N^{+}\left(B_{1} \cup B_{2}\right)$ $=N^{+}\left(B_{1}\right) \cup N^{+}\left(B_{2}\right)$. Hence also $\bigcup_{B \in \mathcal{B}^{\prime}} N^{+}(B) \supseteq S_{0}$ holds and $\mathcal{B}^{\prime}$ has less pairs of crossing fragments than the subset before (for details see Lemma 2 in [17]). Continuing in this way, we get a $\mathcal{C} \subseteq \mathcal{B}$, which does not contain crossing fragments. (An unknown referee pointed out that one can also see that this process ends in a crossing free set of fragments in the following way: in every step $\left|\mathcal{B}^{\prime}\right|$ becomes smaller or $\left|\mathcal{B}^{\prime}\right|$ does not change, but $\sum_{B \in \mathcal{B}^{\prime}}|B|^{2}$ gets bigger.) By the construction of $\mathcal{C}$ using Lemma 4 , we still have $\mathcal{B}_{0} \subseteq \mathcal{C}$ and

$$
\text { (3a) } \bigcup_{C \in \mathcal{C}} N^{+}(C) \supseteq S_{0} \text {. }
$$

Incomparable fragments $C_{1}, C_{2}$ in $(\mathcal{C}, \subseteq)$ are disjoint, since $\mathcal{C}$ is crossing free. 
For the deduction of some properties of $\mathcal{C}$, some further notation is useful. For $C \in \mathcal{C}$ define $b(C):=\left|\mathcal{B}_{0}(C)\right|$ and $\overline{N^{+}}(C):=\bigcup_{C^{\prime} \in \mathcal{C}(C)} N^{+}\left(C^{\prime}\right)$. For (pairwise) incomparable $C_{1}, C_{2}, \ldots, C_{p}$ in $(\mathcal{C}, \subseteq)$ we define the height $h\left(C_{1}, C_{2}, \ldots, C_{p}\right):=$ $\left(\sum_{i=1}^{p}\left|\mathcal{C}\left(C_{i}\right)\right|\right)-p=\sum_{i=1}^{p}\left|\dot{\mathcal{C}}\left(C_{i}\right)\right|$.

(5) Let $C_{1}, C_{2}, \ldots, C_{p}$ be incomparable in $(\mathcal{C}, \subseteq)$.

Then $\left|\bigcup_{i=1}^{p} N^{+}\left(C_{i}\right)-\bigcup_{i=1}^{p} \overline{\mathcal{B}_{0}\left(C_{i}\right)}\right| \geq k$ holds.

Proof. Let be $T:=\bigcup_{i=1}^{p} N^{+}\left(C_{i}\right)$ and $S:=\bigcup_{i=1}^{p} \overline{\mathcal{B}_{0}\left(C_{i}\right)}$. If $|T \cap A| \geq k$ holds, then the inequality in (5) is true, since $A \cap S=\emptyset$. So we may assume that there is an $a \in A-T$, since $|A|>k$. Since $\kappa(D)=k$ and $|S|>k$, there is an $S, a$-fan $P_{1}, \ldots, P_{k}$. Let $b_{i}$ be the first vertex of $P_{i}$. Since $b_{i} \in S$ and the $C_{j}$ are disjoint, there is exactly one $j_{i}$ with $b_{i} \in \overline{\mathcal{B}_{0}\left(C_{j_{i}}\right)} \subseteq C_{j_{i}}$. Since $a \notin T \cup S$, there must be a $t_{i} \in\left(P_{i}-b_{i}\right) \cap N^{+}\left(C_{j_{i}}\right)$. All these $t_{i}$ are distinct, since $t_{i} \neq a$ and the $P_{i}-a(\mathrm{i}=1, \ldots, \mathrm{k})$ are disjoint. Since $\left(P_{i}-b_{i}\right) \cap S=\emptyset$ holds, we conclude $|T-S| \geq k$

(6) Let $C_{1}, \ldots, C_{p}$ be incomparable elements of $(\mathcal{C}, \subseteq)$.

Then $\left|\bigcup_{i=1}^{p} \overline{N^{+}}\left(C_{i}\right) \cap \bigcup_{i=1}^{p} \overline{\mathcal{B}_{0}\left(C_{i}\right)}\right| \leq\left(\left(\sum_{i=1}^{p} b\left(C_{i}\right)\right)-1\right) k$.

Proof. We induct on the height $h\left(C_{1}, \ldots, C_{p}\right)$. Let be $T$ and $S$ as in the last proof.

(a) $h\left(C_{1}, \ldots, C_{p}\right)=0$.

Then we have $C_{i} \in \mathcal{B}_{0}$ for all $\mathrm{i}$ and $\overline{N^{+}}\left(C_{i}\right)=N^{+}\left(C_{i}\right)$ and $\overline{\mathcal{B}_{0}\left(C_{i}\right)}=V\left(C_{i}\right)$ holds for $\mathrm{i}=1, \ldots, \mathrm{p}$. Therefore, (5) implies $\left|\bigcup_{i=1}^{p} \overline{N^{+}}\left(C_{i}\right) \cap S\right|=|T \cap S| \leq p k-k$.

(b) $h\left(C_{1}, \ldots, C_{p}\right)>0$.

We distinguish two cases.

$(\alpha) \mathrm{p}=1$.

Then $C_{1} \notin \mathcal{B}_{0}$. Let $C^{1}, \ldots, C^{q}$ be the distinct maximal elements of $\dot{\mathcal{C}}\left(C_{1}\right) \neq$ $\emptyset$. From $h\left(C_{1}\right)>0$ we get $q \geq 1$. Being maximal elements, $C^{1}, \ldots, C^{q}$ are incomparable w.r.t. $\subseteq$. Since the height of $C^{1}, \ldots, C^{q}$ is less than the height of $C_{1}$, we get by induction $\left|\bigcup_{i=1}^{q} \overline{N^{+}}\left(C^{i}\right) \cap \bigcup_{i=1}^{q} \overline{\mathcal{B}_{0}\left(C^{i}\right)}\right| \leq\left(\left(\sum_{i=1}^{q} b\left(C^{i}\right)\right)-1\right) k$.

Of course, $\bigcup_{i=1}^{q} \overline{\mathcal{B}_{0}\left(C^{i}\right)}=\overline{\mathcal{B}\left(C_{1}\right)}$ and $\sum_{i=1}^{q} b\left(C^{i}\right)=b\left(C_{1}\right)$ hold. But $N^{+}\left(C_{1}\right) \cap$ $\overline{\mathcal{B}_{0}\left(C_{1}\right)}=\emptyset$, since $\overline{\mathcal{B}_{0}\left(C_{1}\right)} \subseteq V\left(C_{1}\right)$. So we get $\left|\overline{N^{+}}\left(C_{1}\right) \cap \overline{\mathcal{B}_{0}\left(C_{1}\right)}\right| \leq\left(b\left(C_{1}\right)-1\right) k$, as claimed.

( $\beta) p \geq 2$.

Now we have $\left|\overline{N^{+}}\left(C_{i}\right) \cap \overline{\mathcal{B}_{0}\left(C_{i}\right)}\right| \leq\left(b\left(C_{i}\right)-1\right) k$ for $\mathrm{i}=1, \ldots, \mathrm{p}$ by $(\alpha)$ or $(\mathrm{a})$. Let us consider a $C_{i}^{\prime} \in \dot{\mathcal{C}}\left(C_{i}\right)$ and $B \in \mathcal{B}_{0}\left(C_{j}\right)$ for $i \neq j$. Let us assume that there is an $x \in N^{+}\left(C_{i}^{\prime}\right) \cap B$. Then $x \in C_{i}$ or $x \in N^{+}\left(C_{i}\right)$, since $C_{i}^{\prime} \subseteq C_{i}$ holds. But $x \in C_{i}$ is not possible, since otherwise $x \in B \cap C_{i} \neq \emptyset$, contradicting $B \subseteq C_{j}$ and $C_{i} \cap C_{j}=\emptyset$. So we conclude $x \in N^{+}\left(C_{i}\right)$. This means $\overline{N^{+}}\left(C_{i}\right) \cap \bigcup_{\substack{j \neq i \\ j=1}}^{p} \overline{\mathcal{B}_{0}\left(C_{j}\right)} \subseteq$ $N^{+}\left(C_{i}\right)$. Therefore, $\overline{N^{+}}\left(C_{i}\right) \cap S \subseteq\left(\overline{N^{+}}\left(C_{i}\right) \cap \overline{\mathcal{B}_{0}\left(C_{i}\right)}\right) \cup N^{+}\left(C_{i}\right)$. Since we know from (5) that at least $k$ elements of $T$ are not contained in $S$, we conclude $\left|\bigcup_{i=1}^{p} \overline{N^{+}}\left(C_{i}\right) \cap S\right| \leq \sum_{i=1}^{p}\left|\overline{N^{+}}\left(C_{i}\right) \cap \overline{\mathcal{B}_{0}\left(C_{i}\right)}\right|+k p-k \leq \sum_{i=1}^{p}\left(b\left(C_{i}\right)-1\right) k+$ 
$k p-k=\sum_{i=1}^{p} b\left(C_{i}\right) k-k$.

Now we easily get a contradiction. Let $C_{1}, \ldots, C_{p}$ be the maximal elements of $(\mathcal{C}, \subseteq)$. Then these are incomparable and an application of $(6)$ gives

(7) $\left|\bigcup_{C \in \mathcal{C}} N^{+}(C) \cap \overline{\mathcal{B}_{0}}\right| \leq\left(\left|\mathcal{B}_{0}\right|-1\right) k$.

On the other hand, from (3a) and (4) follows

$$
\text { (8) }\left|\bigcup_{C \in \mathcal{C}} N^{+}(C) \cap \overline{\mathcal{B}_{0}}\right| \geq\left|S_{0}\right| \geq\left|\mathcal{B}_{0}\right|(k+1)-k .
$$

But (7) and (8) are contradictory

\section{References}

[1] R. Halin, A theorem on n-connected graphs, J. Combin. Theory 7 (1969), $150-154$.

[2] R. Halin, Zur Theorie der n-fach zusammenhängenden Graphen, Abh. Math. Sem. Univ. Hamburg 33 (1969), 133-164.

[3] R. Halin, Untersuchungen über minimale n-fach zusammenhängende Graphen, Math. Ann. 182 (1969), 175 - 188.

[4] R. Halin, Kreise beschränkter Länge in gewissen minimalen n-fach zusammenhängenden Graphen, Math. Ann. 183 (1969), 323 - 327.

[5] R. Halin, Studies on minimally n-connected graphs, pp. 129 - 136 in Combinatorial Mathematics and its Applications, Academic Press, London1971.

[6] R. Halin, Unendliche minimale n-fach zusammenhängende Graphen, Abh. Math. Sem. Univ. Hamburg 36 (1971), 75 - 88.

[7] D. R. Lick, Minimally n-line connected graphs, J. Reine Angew. Math. 252 (1972), 178-182.

[8] G. Chartrand, A. Kaugars, and D. R. Lick, Critically n-connected graphs, Proc. Amer. Math. Soc. 32 (1972), 63 - 68.

[9] T. Kameda, Note on Halin's theorem on minimally connected graphs, J.Combin. Theory (B) 17 (1974), 1-4.

[10] W. Mader, Ecken vom Innen- und Außengrad $n$ in minimal n-fach kantenzusammenhängenden Digraphen, Arch. Math. 25 (1974), 107-112.

[11] Y. O. Hamidoune, Sur les atomes d'un graphe orienté. C. R. Acad. Sci. Paris Sér. A 284 (1977), 1253-1256. 
[12] W. Mader, Connectivity and edge-connectivity in finite graphs, pp. 6695 in Surveys in Combinatorics (ed. B. Bolllobás), Cambridge University Press 1979.

[13] W. Mader, Minimal n-fach zusammenhängende Digraphen, J. Combin. Theory (B) 38 (1985), 102 - 117.

[14] W. Mader, Kritisch n-fach kantenzusammenhängende Graphen, J.Combin. Theory (B) 40 (1986),152-158.

[15] W. Mader, Generalizations of critical connectivity of graphs, Discrete Mathematics 72 (1988), 267 - 283.

[16] W. Mader, On critically connected digraphs, J. Graph Theory 13 (1989), $513-522$

[17] W. Mader, Ecken von kleinem Grad in kritisch n-fach zusammenhängenden Digraphen, J. Combin. Theory (B) 53 (1991), 260-272.

[18] W. Mader, Critically n-connected digraphs, pp. 811- 829 in Graph Theory, Combinatorics, and Applications, Vol.2 (eds. Y. Alavi, G. Chartrand, O. R. Oellermann, A. J. Schwenk), John Wiley and Sons 1991.

[19] W. Mader, On vertices of outdegree $\mathrm{n}$ in minimally n-connected digraphs, J. Graph Theory 39 (2002), 129 - 144.

[20] W. Mader, High connectivity keeping sets in graphs and digraphs, Discrete Math. 302 (2005), 173-187.

[21] W. Mader, Connectivity keeping trees in k-connected graphs, J. Graph Theory 69 (2012), 324 - 329. 